\title{
Bidirectional Coupling of Macroscopic and Microscopic Approaches for Pedestrian Behavior Prediction ${ }^{1}$
}

\author{
Angelika Kneidl, Markus Thiemann, André Borrmann, Stefan Ruzika, Horst \\ W. Hamacher, Gerta Köster, Ernst Rank \\ Technische Universität München, 80290 Munich, Germany \\ Technische Universität Kaiserslautern, 67663 Kaiserslautern, Germany \\ Siemens AG, CT PP2, 80200 Munich, Germany \\ Corresponding author: kneidl@bv.tum.de
}

\begin{abstract}
We combine a macroscopic and a microscopic model of pedestrian dynamics with a bidirectional coupling technique to obtain realistic predictions for evacuation times. While the macroscopic model is derived from dynamic network flow theory, the microscopic model is based on a cellular automaton. Output from each model is fed into the other, thus establishing a control cycle. As a result, the gap between the evacuation times computed by both models is narrowed down: the microscopic approach benefits from route optimization resulting in lower evacuation times. The network flow approach is enriched by including data of microscopic pedestrian behavior, thus reducing the underestimation of evacuation times.
\end{abstract}

\section{Introduction}

Modeling pedestrian dynamics to predict pedestrian behavior for both standard and panic situations has been examined using various approaches, as described in [1]. One goal of modeling pedestrian crowds is to find lower bounds for the evacuation time of a given scenario, e.g. buildings, regions, etc. Network flowbased approaches are capable of yielding this information [2]. However, since some aspects of pedestrian behavior - such as interaction - are not taken into account, the estimated times will probably never be reached in real, nevertheless they can serve as a lower bound for evacuation times.

Our goal is to find a more realistic lower bound. To this end, we propose to combine two different approaches. First, we use a macroscopic model based on quickest flows in dynamic networks to compute optimal routing strategies [3]. Then we apply a microscopic model to capture pedestrian behavior and derive a

1 This research is partly supported by the Federal Ministry for Education and Research (Bundesministerium für Bildung und Forschung, BMBF), Project REPKA, under FKZ 13N9961 (TU Kaiserslautern), 13N9964 (Siemens) 
heuristic upper bound. The model is based on a cellular automaton [4-7] using a potential field to describe forces (according to [1]). The two estimates enfold the true evacuation time like a sandwich [8]. We couple the two approaches by means of a control cycle feeding output from one model into the other, and vice versa.

This article is organized as follows: to begin with, we describe the macroscopic and microscopic models. Then we specify the setup of the bidirectional coupling relating to the two models. Results and an outlook on further research conclude the article.

\section{Description and Setup of the Macroscopic Model (Optimization)}

The scenario (building, region, etc.) is modeled using a discrete-time dynamic network $G=(N, A, T)$, where $N$ is a set of nodes, $A$ is a set of directed arcs, and $T$ is a finite time horizon discretized into the set $\{0, \ldots, T\}$. The node set $N$ subsumes a source $s \mu N$ and a target $t \mu N$. Each arc $(i, j) \mu A$ has an associated timedependent capacity $\left.\left.u_{i j}(\theta) \mu\right]\right]_{0}^{+}$and a time-dependent travel time $\left.\tau_{i j}(\theta) \mu\right]_{0}^{+}$for all time steps $\theta=0, \ldots, T$. Here, $u_{i j}(\theta)$ limits the number of flow units that can enter arc $(i, j)$ at time $\theta$. We assume that the node capacity is zero for all nodes and all time steps, i.e. no waiting at nodes is permitted. The travel time $\tau_{i j}(\theta)$ defines the time needed to traverse $\operatorname{arc}(i, j)$ for flow departing from node $i$ at time $\theta$, i.e. the flow will arrive at node $j$ at time $\theta+\tau_{i j}(\theta)$.

A flow is a function $x: A \times\{0, \ldots, T\} \rightarrow]{ }_{0}^{+}$which assigns a non-negative value to each arc for all time steps and which is subject to flow conservation and capacity constraints. For a more detailed introduction on network flows we refer readers to the book of Ahuja et al. [9].

The goal of the quickest flow problem (see [10]) is to find a feasible flow $x$ which sends a given number of flow units $U \mu]{ }_{0}^{+}$from $s$ to $t$ in the shortest time $T_{U} \leq \mathrm{T}$. With the setting given above, the problem is called discrete-time quickest flow problem with time-dependent attributes. We refer to [11] for mathematical details.

\section{Network Setup for Realizing the Coupling}

To model pedestrian movements using dynamic network flows, we represent corridors, walkways, streets etc. in a given scenario as arcs in the network. Every $\operatorname{arc}(i, j) \mu A$ has a corresponding fixed width $w_{i j}[m]$ and length $l_{i j}[m]$. In the coupling setup, we predefine the maximum possible rate of flow per unit width $M_{i j}[$ peds $/ m s]$ for every arc $(i, j) \mu A$, henceforth called the specific flow rate of arc $(i, j)$. We fix the length of the basic time unit for the network parameters as $z=1 s$. Based on this data we compute the capacity as $u_{i j}=\left\lfloor M_{i j} \cdot w_{i j} \cdot 1 / z\right\rfloor$. Note that the capacity is constant over time. Moreover, an average velocity $v_{i j}(\theta)$ for every arc $(i, j) \mu A$ and $\theta=\{0, \ldots, T\}$ with corresponding travel time $\tau_{i j}(\theta)=\left\lfloor l_{i j} \cdot v_{i j}(\theta) \cdot z\right\rfloor$ is assumed to be known. 


\section{Description and Setup of the Microscopic Model (Simulation)}

Our microscopic model is based on a cellular automaton [4-7]. The whole area of interest is discretized by hexagonal cells, each of which can accommodate an average European male [12]. At each time step, each cell can be occupied either by a pedestrian, an obstacle, a source or a target. Pedestrians move according to specific behavior rules from sources to targets. The movement of a pedestrian is influenced by different forces, namely the repellent forces of obstacles and other pedestrians and the attraction of the targets. All forces are represented by a common potential field. At each time step, each person moves to an accessible neighboring cell with minimum potential field value. Once the target has been reached, the person vanishes from the model. Each pedestrian is "born" with a certain desired walking speed - the so-called free flow velocity [1,12]. Depending on the local density, i.e. the number of pedestrians in the surrounding cells, pedestrians are forced to slow down. The code is calibrated in such a way as to reproduce Weidmann's fundamental diagram [12]. For a more detailed description of the microscopic model, please refer to [13].

\section{Extensions of the Model for Realizing the Coupling}

For the coupling, we define a graph on top of the cellular automaton that is automatically derived from the underlying topography - this is done by finding orientation points on the bisector of each convex obstacle corner (see Fig. 1). These orientation points refer to graph nodes and each point is subsequently connected to all orientation points in sight by means of an arc. In addition, they are connected to the source and the target in the same manner. This graph, including the arc parameters width and length, is used in the macroscopic setup to construct the network. In the microscopic model we use this graph to replace the target and obstacle function of our potential.

In the simulation, a pedestrian traverses along arcs of the graph leading to his target. The macroscopic model yields a distribution rate for each node, according to which the pedestrians choose their next intermediate target.

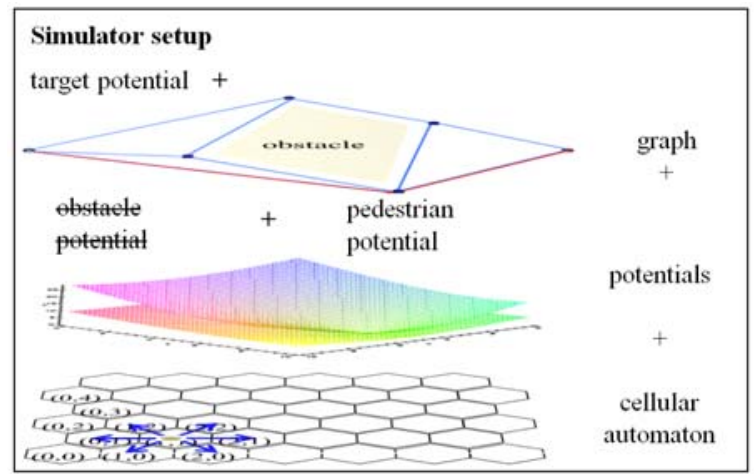

Fig. 1 Microscopic setup 


\section{Control Cycle Setup and Constraints for Realizing the Coupling}

We define shared (fixed) parameters and variable parameters, which are adapted in each control cycle. A cycle consists of one optimization run followed by one simulation run.

\begin{tabular}{ll}
\hline Shared (fixed) parameters & Variable parameters \\
\hline $\begin{array}{l}\text { Scenario including network derived with arc } \\
\text { width and length }\end{array}$ & $\begin{array}{l}\text { Distribution ratios for each arc and each time } \\
\text { step }\end{array}$ \\
Number of pedestrians & Source flow quantity for each time step \\
Time step size & Average velocity on each arc for each time step \\
Specific flow rate for all arcs & \\
\hline
\end{tabular}

Scenario including the network derived - We choose different scenarios to test our method. Each scenario consists of one source and one final target plus some intermediate targets and the derived network.

Number of pedestrians - The number of pedestrians has to be large enough to observe interaction between the two models.

Time step size - The time step size describes the common interval size of the parameter exchange between the two models. In each time step the values are averaged and adapted by both models, respectively.

Specific flow rate (SFR) for each arc - The SFR on an arc corresponds to the maximum number of pedestrians who can move through unit width in one second along that arc. We determine the SFR for each arc within the simulation in a preprocessing phase.

Source flow quantity (SFQ) for each time step - The source flow quantity is a result of the quickest flow calculated in the optimization network. For each time step, the amount of flow leaving the source in the network defines the number of pedestrians to be generated in the corresponding time step of the simulation run. Interaction between pedestrians may prevent the creation of all required pedestrians in a single time step. In this case, they are generated in the subsequent time step.

The number of effectively generated pedestrians is fed back to the optimization to serve as a reference. In our tests we consider two cases: one with feedback and adaptation of the flow quantities by the optimization, and one without. The adaptation of the flow quantity within the optimization works as follows: if the optimal flow quantity is not achieved in the simulation in a single time step, then the overall capacity of the source (i.e. the total amount of flow that can be sent from the source) in this time step is reduced to the smaller value from the simulation. 
Time-dependent distribution ratios for each arc - The second output of the optimization is the time-dependent flow distribution ratio on each arc incident to some node. The distribution ratios are calculated as an average value at each time step. The pedestrians are distributed according to these ratios at the corresponding orientation points during the simulation.

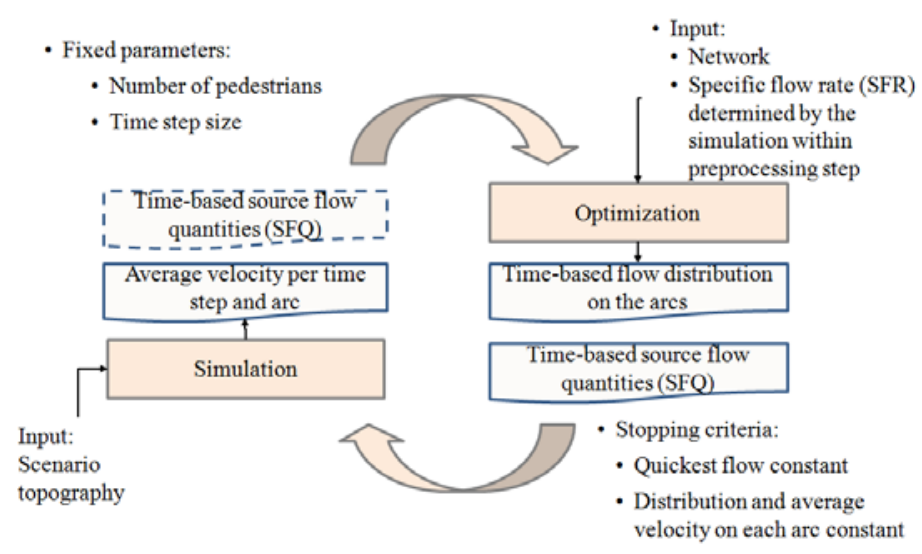

Fig. 2 Coupling setup

Average velocity on each arc for each time step - The simulation returns the average velocities for each arc and time step.

In coupling cycle $i \mu\{1, \ldots$, number of coupling cycles $\}$, these velocities are read in by the optimization in the following manner:

$$
v_{\text {regulation }}^{i}=\alpha v^{i}+(1-\alpha) v_{\text {regulation }}^{i-1} \text {. }
$$

Here, $v_{\text {regulation }}^{0}$ is the velocity used in the initial dynamic network of cycle zero. The parameter $\alpha \in[0,1]$ refers to the predefined weight of the new average velocities.

Fig. 2 gives an overview of all parameters and the way they are exchanged within one coupling cycle.

\section{The Control Cycle}

Before the actual control cycle starts, the SFR is derived on each arc of the network by means of a pre-processing phase, as described above. The initial dynamic network is currently established with the arc parameters (width and length) and, for simplicity, an average walking speed of $1.34 \mathrm{~m} / \mathrm{s}$ for all pedestrians, as in [12]. The quickest flow is computed in this network. The corresponding time-dependent flow distribution ratios on each arc and the timedependent flow quantity of the source are returned as input parameters for the simulation. The simulation sends the pedestrians from the source towards the target according to these two variable parameters. We get time-dependent average 
walking speeds on each arc as a result. The time-dependent travel times of the arcs in the dynamic network are adjusted on the basis of these average velocities. The quickest flow is computed in the modified network, the source quantities and flow distributions are updated and, once again, returned to the simulation. This cycle is repeated for a fixed number of times or until a stopping criterion is satisfied.

\section{The Results of the Bidirectional Coupling}

\section{Choice of Scenario}

We tested the coupling in several scenarios. In this article, we present results for one representative scenario, combining different effects observed during the testing of the coupling. We consider a triangular walkway in combination with a bottleneck. The topography used in the simulation and the corresponding optimization network with arc parameters width and length are given in Fig. 3. There are two points of special interest in the scenario - the junction at $a$ and the bottleneck $b$.

(a)

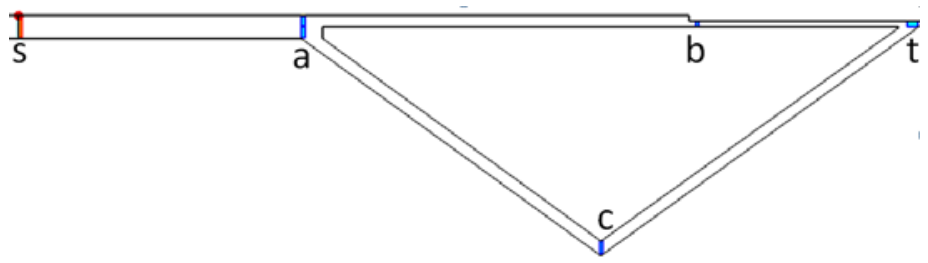

(b)

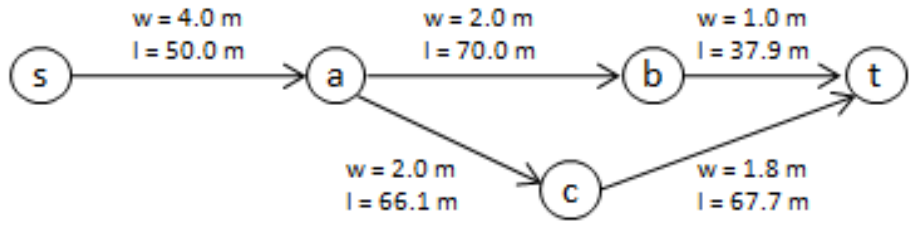

Fig. 3 Topography of example scenario (a) and corresponding network (b)

\section{Parameter Variation}

We investigate the scenario with various parameter configurations.

The first varying parameter is the source flow quantity (SFQ) feedback of the simulation. Secondly, we adjusted the time step size - we contemplate the results for 5 and 10 seconds.

\begin{tabular}{lll}
\hline Configuration & SFQ feedback & Time step size (s) \\
\hline Config_a & no & 10 \\
Config_b & yes & 10 \\
Config_c & no & 5 \\
Config_d & yes & 5 \\
\hline
\end{tabular}

Table 1 List of tested configurations 


\begin{tabular}{ll}
\hline Parameter & Value \\
\hline No. of pedestrians & 1,000 \\
$\alpha$ & 0.3 \\
Initially assumed velocity & $1.34 \mathrm{~m} / \mathrm{s}$ \\
Number of coupling cycles & 25 \\
SFR & Computed in preprocessing phase \\
\hline
\end{tabular}

Table 2 Parameter overview

The different configurations are summarized in Table 1 . Table 2 shows all other parameters which are left unchanged for all configurations.

\section{Results}

The results for the different configurations are summarized in Fig. 4. The curves show the time of the quickest flow obtained by network flow optimization compared to the egress time computed by the simulation. For the simulation we define as egress time the moment when $99 \%$ of the pedestrians have reached the target. In the simulation, pedestrians may be diverted from their original path onto the secondary path in a dense crowd. Not yet having implemented personal strategy changes, they must resume their original path after it has been cleared. Thus they become extreme latecomers. Without the $99 \%$ rule, they would distort the results. In the following, we refer to the egress time as simulation time. Simulation times are plotted for each cycle starting with the values of "cycle zero" showing the output of both models without coupling. We ran 25 cycles to get a representative statement.

Fig. 4 shows a steady increase of the quickest flow in all four configurations during the first few cycles of the coupling. In the remaining cycles it oscillates around a steady state. The simulation time, on the other hand, decreases from its initial value to approach a steady state from above. Again, we observe the typical oscillations of a control cycle. In this case, the oscillations are caused by the difficulty of controlling the pedestrians in front of the junction $a$. A slightly different distribution at node $a$ may result in slightly more or less congestion at the junction and therefore in an increased or reduced total egress time, respectively.

The steady states enclose a small corridor. The only exception is Config_d, where the time step size of 5 seconds is too small to avoid disturbing fluctuations in the gliding average values for the feedback circle. We observe an approximation of both simulation times with a certain overshooting of the lower estimate. The results suggest that, with a sufficiently small time step, it is possible to achieve the convergence of the two approaches to a common steady state. In practice, this is restricted by the fact that meaningful average values can only be obtained with a sufficiently large time step size. But even without convergence, we achieve a substantial improvement of the evacuation time estimates for both approaches. 


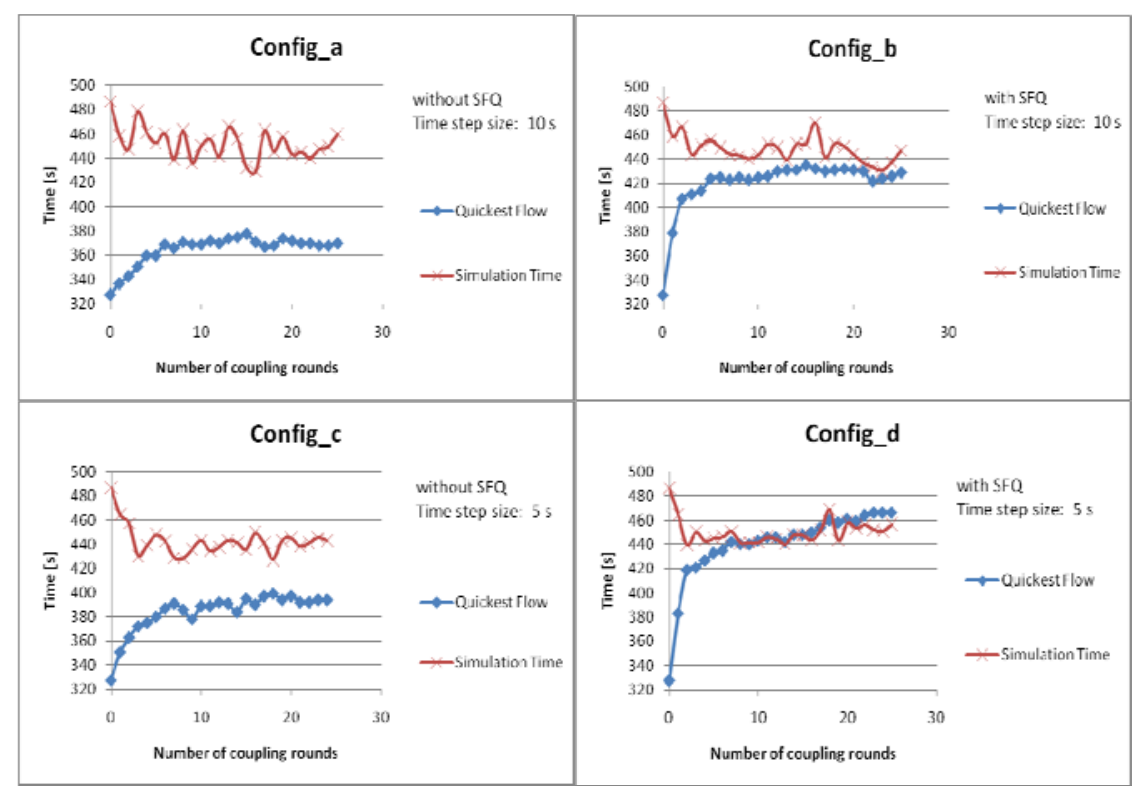

Fig. 4 Coupling results for scenario of Fig. 3 comparing the quickest flow and simulation time for Config_a (top left), Config_b (top right), Config_c (bottom left), Config_d (bottom right)

The incremental value of the quickest flow results from increasing travel times on the arcs of the network induced by the simulation feedback: the simulation captures pedestrian interaction which slows down the crowd. It returns a realistic average velocity below the optimal average velocity to the flow model.

The decreasing value of the simulation is caused by both the adapted source flow quantity (SFQ) in the simulation and the adapted distribution ratio at the node $a$ : In the stand-alone simulation run, all pedestrians take the shortest path from $a$ via $b$ to $t$. The adaptation of the distribution ratio from the optimal flow ensures that, for certain time steps, a given percentage of the pedestrians deviates to the longer route from $a$ via $c$ to $t$. This results in less congestion on the original path, especially in front of the bottleneck $b$, and the pedestrians move faster. After a few coupling cycles, the last individuals in the pedestrian streams on the two paths from $a$ to $t$ reach the target almost simultaneously. This is exactly what one expects from an optimal flow in a network.

The effect of the SFQ is as follows: the capacities on the arcs of the network are bounded by the maximum possible specific flow rate (SFR) in the simulation. Since every feasible flow in the dynamic network maintains the SFR on every arc, congestion is averted. By adapting the value for the SFQ in each time step and sending the pedestrians along paths corresponding to the optimal flow, the simulation similarly maintains the SFR on every arc for all time steps, thus preventing congestion (especially in front of the bottlenecks). This in turn reduces the total time needed for all pedestrians to reach the target. 
Comparing Config_a and Config_b in Fig. 4 shows the positive influence of the SFQ feedback. For a time step size of ten seconds, near-convergence of the steady states of the two approaches is reached with the SFQ feedback (Config_b). The SFQ feedback reduces network capacities whenever the simulation is unable to actually generate the number of pedestrians suggested by the flow model. Otherwise the two models tend to decouple and converge to their separate steady states.

Config_c and Config_d in Fig. 4 show the same results for a time step size of five seconds with the overshooting due to fluctuations in the average velocity values. We describe one cause of the overshooting: Very small average velocities derived from a short period of observing congestion induce smaller source flow quantities on the optimization side in the next cycles. While these velocities rise again in the next coupling cycle (because no congestion occurs), the source quantities will not increase due to our definition of how the SFQ values are handled. This leads to a capacity limit that is too strict, hence the value of the quickest flow increases. To summarize, both parameters influence each other very sensitively and can therefore induce a chain reaction causing an overshooting of the lower steady state.

The bidirectional coupling leads to an improvement in the evacuation time estimate for both models. Using parameter variation we are able to adjust the interaction sensitivity of the two models during the control cycle. However, parameters have to be adapted with caution. For the given scenario, the optimization curve approximates the simulation curve best with the adapted SFQ feedback.

\section{Outlook}

We have presented a control cycle to combine a macroscopic and a microscopic approach for modeling pedestrian behavior. The coupling leads to a modification of the dynamic network so that the computed quickest flow is almost reproducible by the simulation. At the same time, the total egress time of the pedestrians in the simulation is lowered due to the source flow quantity and flow distribution given by the optimization.

For the given example, the egress times computed by both models approach steady states. The steady states enclose a small corridor. This effect could also be observed for other scenarios. The results of the simple scenario suggest that, with the right choice of parameters, the gap can almost be closed.

We propose to continue our work by testing the control cycle for bigger scenarios with more junctions and bottlenecks to answer questions such as: Will the results remain as smooth as in the small examples? Will it be possible to close the gap between the two models in a more complex setup? Also, in the experiments presented here, all pedestrians share the same free flow velocity. This is 
unrealistic. In a follow-up step, we will assign individual free flow velocities through a Gauss distribution, as described in [12] and observe the effects on the control cycle.

\section{References}

1. Schadschneider, A., Klingsch, W., Kluepfel, H., Kretz, T., Rogsch, C., and Seyfried, A.: Evacuation Dynamics: Empirical results, Modeling and Applications. In: R.A. Meyers (Ed.), Encyclopedia of Complexity and System Science Vol. 3 (pp. 3142-3176), Springer, Berlin Heidelberg (2009)

2. Chalmet, L.G., Francis, R.L., and Saunders, P.B.: Network Models for Building Evacuation, Management Science, 28, 86-105 (1982)

3. Hamacher, H.W. and Tjandra, S.A.: Mathematical Modelling of Evacuation Problems: A State of the Art. In: M. Schreckenberger and S.D. Sharma (Eds.), Pedestrian and Evacuation Dynamics (pp. 227-266), Springer, Berlin Heidelberg (2002)

4. Emmerich, H. and Rank, E.: An Improved Cellular Automaton Model for Traffic Flow Simulation, Physica A, 234, pp. 676-686, (1997)

5. Burstedde, C., Klauck, K., Schadschneider, A., and Zittartz, J.: Simulation of Pedestrian Dynamics Using a 2-dimensional Cellular Automaton. Physica A, 295, 507-525 (2001)

6. Kinkeldey, C.: Fussgängersimulation auf der Basis zellulärer Automaten: Studienarbeit im Fach Bauinformatik, Universität Hannover (2003)

7. TraffGo Handbuch: www.traffgoht.com/de/pedestrians/downloads/index.html

8. Hamacher, H.W., Heller, S., Klein, W., Köster, G., and Ruzika, S.: A Sandwich Approach for Evacuation Time Bounds. to appear in conference proceedings PED 2010

9. Ahuja, R.K., Magnanti, T.L., and Orlin, J.B.: Network Flows: Theory, Algorithms, and Applications, Prentice Hall, Englewood Cliffs, New Jersey (1993)

10. Burkard, R., Dlaska, K., and Klinz, B.: The Quickest Flow Problem, ZOR Methods and Models of Operations Research, 37, 31-58 (1993)

11. Tjandra, S.A.: Dynamic Network Optimization with Application to the Evacuation Problem, PhD thesis, Universität Kaiserslautern, Shaker, Aachen (2003)

12. Weidmann U.: Transporttechnik der Fussgänger, Schriftenreihe des IVT 90, Zürich (1992)

13. Klein, W., Köster, G., and Meister, A.: Towards the Calibration of Pedestrian Stream Models, Lecture Notes in Computer Science: PPAM 2009, Springer, Berlin Heidelberg (2010) 